\title{
Analysis on the Influence Factors of Construction Linear Control of Continuous Rigid Structure Bridge
}

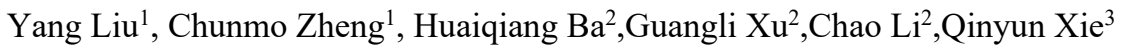 \\ ${ }^{1}$ School of Architecture and Planning, Yunnan University, Kunming 650091, China \\ ${ }^{2}$ Yunnan Kunchu expressway investment and Development Co., Ltd, Kunming 650030, China \\ ${ }^{3}$ Yunnan Dazhu Technology Co., Ltd, Kunming 650030, China
}

\begin{abstract}
In order to study the influence of prestress on cantilever deflection and construction linear control of continuous rigid frame bridge in construction stage, this paper introduces the significance of continuous rigid frame bridge's linear control, the calculation principle and deflection influence analysis of vertical formwork elevation in cantilever construction. According to a specific continuous rigid frame bridge, this paper use the finite element software to simulate and calculate the deflection of prestress to the cantilever construction of continuous rigid frame bridge. The influence of friction coefficient between prestressed steel bundle and bellows and prestress loss on cantilever deflection and construction line control of continuous rigid frame bridge is also analyzed, furtherly brings out the solution to deal with the problems due to the change of prestress.
\end{abstract}

\section{Introduction}

The prestressed tendons are tensioned before the combination of concrete, so as to change the working state of concrete, put pressure on concrete in advance, making the high strength steel bar play a full role, also make the concrete's compressive performance better. Meanwhile, restressing the concrete members in advance can offset part of the used load or even all because of the direction of action is exactly opposite to the direction of the used load. When the torque generated by the external action on the beam section is offset by the torque generated by the applied prestress, the flexural member can be designed as a compression member, thus improving the mechanical performance of the concrete member. The prestressed continuous rigid frame bridge has the advantages of large stiffness, small deformation, less expansion joint, good driving comfort, high integrity and high seismic performance. ${ }^{[2]}$ In the construction of prestressed rigid frame bridge, the cantilever construction is represented by the hanging basket construction. The two adjacent piers move the hanging basket to both sides at the same time, advancing horizontally and synchronously. The suspension is connected with the long symmetrical construction section by section along the span direction until the span is closed, and the beam sections are tightly connected to the whole by tensioning prestressed steel bundles. Under the action of prestress, the lower deflection brought by each construction stage can be offset. By controlling the prestressing tendons, the reverse deflection is produced, and the concrete is pre-loaded, thus offsetting the lower deflection brought by the later use of the load, Meet the design requirements. Therefore, the linear control of continuous rigid frame by prestress is the main factor.

\section{Continuous rigid frame construction control}

In order to ensure the construction quality of the bridge and make the structure internal force and displacement meet the design requirements, it is necessary to control the construction of prestressed concrete continuous rigid frame bridge, Includes deformation control and internal force control. Deformation control is mainly to strictly control the vertical deflection and transverse deviation of each section of box girder. When there is a deviation and the deviation is large, the error analysis is carried out immediately and the adjustment scheme is made to prepare for the more accurate construction of the next section. Because the structural state of continuous rigid frame can not be adjusted after construction, prediction control is mainly used in construction control, including construction control structure simulation analysis, construction monitoring, construction error analysis and subsequent state prediction, etc[1].

\section{Analysis of deflection and calculation principle of vertical die elevation}

\subsection{Deflection analysis}

In the actual construction, there is often a situation that does not accord with the actual design, and the actual situation deviates from the theoretical calculation. For 
continuous rigid frame, deflection control is the basis of elevation control. In order to judge whether the beam force and deformation are normal and whether the theoretical calculation model is in line with the reality. The deflection of continuous rigid frame bridge during construction includes beam weight, pre-stress, concrete shrinkage and creep, and hanging basket deformation. Deflection calculation mainly includes: a. deflection and cumulative deflection of each node section in each construction stage. b. the cumulative deflection of each node section in the bridge state. c. maximum (minimum) live load deflection of each node section[3].

\subsubsection{Calculation of deflection of dead load, live load and prestress}

The deflection of cantilever beam with variable cross section is calculated by conjugate beam method, and the bending moment $\mathrm{M}$ generated by dead load, live load and prestress on virtual beam can be obtained X arbitrary sectionjDeflection at:

$$
f_{j}=\sum_{i=1}^{j} \frac{M_{i}}{E_{i} I_{i}}\left(x_{i}-\xi_{i}\right) d_{i} \quad(\mathrm{i}<\mathrm{j})
$$

$\mathrm{M}_{\mathrm{i}}$ : Average bending moment of section i beam

$\mathrm{I}_{\mathrm{i}}$ : i moment of inertia of section of beam section

\subsubsection{Calculation of shrinkage creep deflection}

At present, the code "Design Code for Highway Reinforced Concrete and Prestressed Concrete Bridges and culverts "(JTG3362-2018) is used to calculate.

\subsection{Pre-arch setting}

In order to make continuous rigid frame under the influence of comprehensive deflection such as dead load, live load, prestress load and concrete creep, it is necessary to set pre-arch for continuous rigid frame during construction, so as to offset the deformation caused by dead weight of bridge structure, bridge deck pavement and railing. The general value is equal to the vertical deflection of all dead loads and half of dead loads.

$f_{\text {camber }}=\left(-f_{y}+f_{g 1}+f_{g 2}\right)(1+\phi(\infty, \tau))+\frac{1}{2} f_{p}$

$\mathrm{f}_{\mathrm{y}}$ : deflection resulting from prestress

$f_{g 1}$ : Self-weight, temporary load and deflection of hanging basket in first stage dead load beam section

$\mathrm{f}_{\mathrm{g} 2}$ : Deflection of secondary dead loads, guardrails and paving

Deflection resulting from $\mathrm{fp}$ : live loads

$\phi(\infty, \tau)$ Final value of shrinkage creep of concrete

\subsection{Calculation Principle of Vertical Model Elevation}

Elevation control is the basic guarantee to ensure the smooth closure of continuous rigid frame, and it is also the decisive factor to ensure that the bridge alignment meets the design requirements. The deflection of each stage in cantilever pouring construction is inevitable, so the elevation of vertical formwork is based on the deflection of each stage, and the pre-arch degree is set in the construction process. The deflection deformation caused by self-weight and other loads is offset by the elevation of vertical die in each stage.

\section{Prestressed deflection analysis}

\subsection{Upper deflection due to prestress}

Because of the characteristics of prestress, the tension of longitudinal prestressed tendons in the stage of construction will produce upward negative deflection, which can effectively offset the deflection caused by other loads, such as self-weight, and is the key to linear control[5].

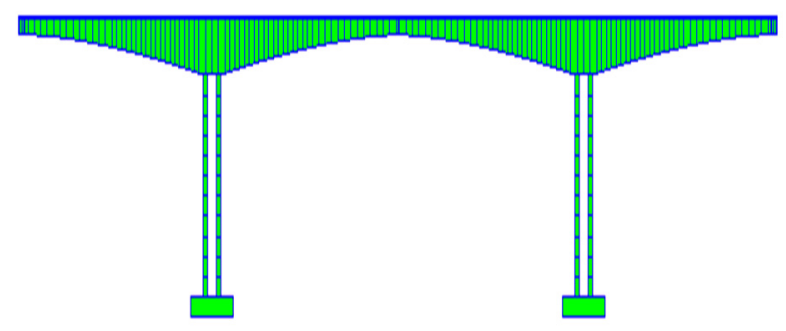

Fig.3.1 Midas civil full bridge model.

Taking prestressed concrete continuous rigid frame bridge $(120 \mathrm{~m}+230 \mathrm{~m}+120 \mathrm{~m})$ as an example, the highway-class I, double two-way four-lane, single bridge width $13.85 \mathrm{~m}$, and double thin-walled pier height $55 \mathrm{~m}$, are modeled by finite element Midas Civil software. The longitudinal prestressed steel bar of the main beam of the bridge is constructed by high strength and low relaxation rate steel strand and $\mathrm{fpk}=1860 \mathrm{Mpa}, \mathrm{E}=1.95 * 105 \mathrm{Mpa}$, using hanging basket cantilever pouring construction, the whole bridge main beam construction has 33 construction stages, according to the actual construction steps to establish the whole bridge model analysis.

According to the construction model of the whole bridge, two working conditions are established, namely, normal prestressed concrete reinforced structure and ordinary prestressed concrete reinforced structure. The deflection deformation curve before the closure is obtained by calculation. Through two kinds of cumulative deflection deformation, it can be clearly found that the cumulative deflection of ordinary concrete reinforced structure in the construction stage is positive deflection, and the vertical deformation is large. Negative deflection can be offset by the deflection of the structure itself, more in line with the design line. The maximum difference in the cumulative deflection between the two is near the maximum cantilever section. The deflection difference at the maximum cantilever is shown in Table 3-1 as below.

Table.3.1 Cumulative deflection difference at maximum cantilever.

\begin{tabular}{ccccc}
\hline Node Number & 12 & 13 & 70 & 71 \\
\hline $\begin{array}{c}\text { Common concrete } \\
\text { structures }(\mathrm{cm})\end{array}$ & -25.43 & -25.77 & -19.04 & -18.06 \\
$\begin{array}{c}\text { prestressed concrete } \\
\text { structures }(\mathrm{cm})\end{array}$ & -5.8 & -5.8 & 1.94 & 4.3
\end{tabular}



Difference $(\mathrm{cm})$
19.63
19.97
20.98

Fig .3.4 Comparison of cumulative deflections before closure.

Through the deflection contrast curve, it is clearly found that the deflection difference between the two is small at block 0 . With the increase of segment construction, the cumulative deflection becomes larger and larger. At this time, the deformation of concrete stiffness becomes smaller and more stable, which provides favorable conditions for the closure stage. By comparing the slope of the curve, it can be concluded that for continuous rigid frame, prestress has the main influence on the construction line control. Under the action of prestress, the relative deflection difference of each stage is small, which is convenient for the actual construction line control. More in line with the design line.

\subsection{Analysis of pipe friction parameters on deflection}

At present, the prestressing continuous rigid frame generally adopts the construction technology of tensioning at both ends of the post-tensioning method. Because of the characteristics of the construction, corrugated pipes are reserved in the box girder in advance, and the pipeline positioning is not accurate during the construction process. The negative friction caused by steel bundle tension and hole wall slip will produce greater friction loss for bending prestressing tensioning[6]According to the new code, the loss of prestress friction caused by pipeline parameters is based on the formula:

$$
\sigma=\sigma_{c o n}\left(1-e^{-(\mu \theta+k x)}\right)
$$

$\sigma_{c o n}$ tensile stress at the end of tension

$\mu$ Friction coefficient between prestressed steel bundle and hole

$\mathrm{k}$ : local deviation coefficient per meter length

$\theta$ : The sum of the bending angles from the end of the tension to the channel of the calculated section

$\mathrm{x}$ : the length of the channel from the tension end to the calculated section

$\mu \mathrm{By}$ analyzing the influence of the friction coefficient and $\mathrm{k}$ on the deflection of the continuous rigid frame, the friction coefficient of the channel is analyzed by using five cases.

Working conditions $1: \mathrm{k}=0.0015=0.17 \mu$

Working conditions 2 : $\mathrm{k}=0.002=0.17 \mu$

Working conditions $3: \mathrm{k}=0.002=0.35 \mu$

Working conditions $4: \mathrm{k}=0.003=0.35 \mu$

Working conditions $5: \mathrm{k}=0.0015=0.15 \mu$

The deflection curve of the main beam can be seen that the deflection of the main beam becomes larger when the $\mu$ is increased, and the deflection of the main beam is relatively larger when the $\mathrm{k}$ is increased. Therefore , $\mu$ friction coefficient and deviation coefficient of prestressed pipe will cause prestress loss and increase vertical deflection of main beam. The reverse deflection changes caused by prestressing forces under different friction parameters of the main sections are worthy of Table 3-2 below.
Table.3.2 Reverse Deflection Value of Prestressed under Section Corresponding to Friction Parameters.

\begin{tabular}{ccccc}
\hline $\begin{array}{c}\text { Deflection } \\
\text { value }(\mathrm{mm})\end{array}$ & $\begin{array}{c}\mathrm{k}=0.002 \\
\mu=0.35\end{array}$ & $\begin{array}{c}\mathrm{k}=0.002 \\
\mu=0.17\end{array}$ & $\begin{array}{c}\mathrm{k}=0.0015 \\
\mu=0.17\end{array}$ & $\begin{array}{c}\mathrm{k}=0.003 \\
\mu=0.35\end{array}$ \\
\hline Section 6 & 0.805 & 0.815 & 0.817 & 0.802 \\
Section 10 & 1.481 & 1.538 & 1.543 & 1.473 \\
Section 14 & 2.604 & 2.613 & 2.619 & 2.594 \\
Section 18 & 5.788 & 5.844 & 5.857 & 5.763 \\
Section 22 & 11.828 & 11.927 & 11.962 & 11.766 \\
Section 26 & 19.437 & 19.533 & 19.553 & 19.408 \\
Section 30 & 35.715 & 35.838 & 35.914 & 35.587 \\
\hline
\end{tabular}

Through the analysis of the reverse deflection value caused by prestress under different friction parameters of the hole, it can be concluded that the deflection value of prestress under different friction coefficients of the same section is affected by friction coefficient. When the $\mu, \mathrm{k}$ increases, the deflection value of prestress becomes smaller; when the $\mathrm{k}$ remains unchanged, when the $\mu$ increases, the deflection value of prestress becomes smaller; when the $\mu$ remains unchanged, when the $\mathrm{k}$ increases, the deflection value of prestress becomes smaller; when the friction coefficient increases, the loss of prestress increases, the reverse deflection value of prestress becomes smaller, and the vertical deflection of main beam becomes larger.

\subsection{Change in deflection due to loss of prestress}

Long-span continuous rigid frame bridge deflection is a prominent disease, among which the loss of prestress is one of the main reasons. Because of the linear control and deflection control of continuous rigid frame, the reverse deflection of prestress is mainly used to offset the deflection caused by the structure itself and long-term use. The loss of prestress is affected by construction method, material and temperature [7]. To study the deflection changes resulting from the loss of prestress, Take the state river bridge, Drawing $1395 \mathrm{MPa}$, at both ends of posttensioning method used in actual construction The finite element model, Under the conditions of $10 \%, 20 \%, 30 \%$ and all prestress loss, respectively, the side span closure (section number 1), the side span 3/4(section number 2), the side span $1 / 2$ (section number 3 ), the side span block 0 (section number 4 ), the middle span $1 / 2$ (section number 5), the middle span 3/4(section number 6), the middle span closure (section number 7) are selected, Vertical deflection of main beam. By comparison, it can be clearly concluded that with the increase of prestress loss, the lower deflection of the main beam is increasing, and the loss of prestress has a significant effect on the alignment of continuous rigid frame bridges. In the same section, with the increase of prestress loss, the vertical deflection of the main beam is $4.71 \mathrm{~cm}$. It can be seen from the chart that the greater the loss of prestress, the greater the deflection difference, which is consistent with the lower deflection of the main 
beam.

\section{Response measures under prestress}

Based on the theoretical analysis of the influence of the deflection of continuous rigid frame on the linear control in the construction stage, combined with the actual project list of the Zhouhe Bridge, the measures to deal with the change of prestress on the linear control are summarized:

(1) Because the reverse deflection caused by prestress is the main factor in the linear control of continuous rigid frame, it is necessary to configure the reasonable number of prestressed tendons according to the bridge type at the beginning of the continuous rigid frame design. Ensure that the line shape after tensioning prestressing bundle is more in line with the design line.

(2) The post-tensioning method is usually used in the construction of cantilever casting. The friction coefficient of the hole $\mathrm{k} 、 \mu$ need to be tested, the friction coefficient between the prestressed steel bundle and the hole is measured $\mu$ and whether the local deviation coefficient per meter length of the hole $\mathrm{k}$, meet the design requirements. If the experimental results are abnormal, the reasons should be found in time to solve the problem[9]The value of $\mathrm{k} 、 \mu$ is $0.001 \sim 0.003$ and $0.1 \sim 0.35$ according to the Design Code for Highway Reinforced Concrete and Prestressed Concrete Bridges and culverts.

(3) The loss of prestress is very important to the linear control of continuous rigid frame bridge, which affects the linear change in the construction stage and the long-term use of the bridge. In the process of tensioning prestressed steel bundles, it is necessary to stretch accurately, to overtension in the design code, to use double control of tension and extension, to predict the loss of prestress, and to monitor the line shape according to the actual construction stage. Adjust the elevation of the model accordingly.

\section{Concluding remarks}

Through the above calculation and analysis, prestress is the main influencing factor of linear control of long span prestressed continuous rigid frame.

(1) Because the reverse deflection brought by prestress significantly changes the deflection value of the main beam and makes the whole bridge more in line with the design line, the monitoring of prestress is the key to the control of the whole line.

(2) Under the action of prestress, the pre-arch should be set according to the actual situation. Because of the deviation between the finite element simulation and the actual situation, the elevation of the vertical die should be corrected in real time.

(3) Because the control of alignment is affected by many factors, it is necessary to combine the prestress comprehensive consideration, use the mathematical statistical method to combine the influencing factors of alignment, select the most unfavorable factors, and carry out linear prediction to ensure safety.
(4) In the field line monitoring, to real-time deflection measurement, timely feedback.

\section{References}

1. YongXia Dong. Shijiazhuang Tiedao University (2013)

2. Yugen Chen. Southeast University (2016)

3. Jiangang Tong. Southwest Jiaotong University (2007)

4. Jiyao Zhang. Cantilever Prestressed Concrete Continuous Beam Bridge (2004)

5. Jianli Pang. Fujian Building Materials 04, 4 (2013)

6. Huabin Fang, Qixian Tian. Bridge Construction 02, 81 (2006)

7. Jianguo Shao. Lanzhou Jiaotong University (2012)

8. Hongchun Han. Southwest Jiaotong University (2007)

9. Jian Huo. Northeast Forestry University (2013) 